\title{
Multiplexed Quantification of Metabolites with MISSILE
}

\section{Jones $\mathrm{DR}^{1 *}$, Wang $\mathrm{X}^{2}$, Shaw $\mathrm{T}^{2,3}$, Cho $\mathrm{JH}^{2}$ and Peng $\mathrm{J}^{2-5 *}$}

${ }^{1}$ Department of Biochemistry and Molecular Pharmacology, Langone Medical Center, New York University, NY, 10016, USA

${ }^{2}$ St. Jude Proteomics Facility, St. Jude Children's Research Hospital, Memphis, TN 38105, USA

${ }^{3}$ Department of Computational Biology, St. Jude Children's Research Hospital, Memphis, TN 38105, USA

${ }^{4}$ Department of Developmental Neurobiology, St. Jude Children's Research Hospital, Memphis, TN 38105, USA

${ }^{5}$ Department of Structural Biology, St. Jude Children's Research Hospital, Memphis, TN 38105, USA

\begin{abstract}
We tested a strategy for multiplexed (4-plex) quantification of metabolites using the MISSILE identification method with liquid chromatography coupled to tandem mass spectrometry. We applied this methodology to study the metabolic effect of the proteasome inhibitor and chemotherapeutic drug Bortezomib in yeast cells. Using JUMPm software version 1.1 we simultaneously identified and quantified 95 metabolites across four experimental conditions and found that Bortezomib increased the accumulation of dipeptides but decreased the levels of specific lipid molecules (e.g. phosphtidylethanolamines) in a dose-dependent manner. This method combines metabolite identification and quantification, making untargeted metabolomics experiments more informative.
\end{abstract}

Keywords: Metabolomics; Metabolome; Mass spectrometry; Stable isotope labeling; Liquid chromatography; Metabolite quantification; Multiplex; Bortezomib; Proteasome inhibition; MISSILE

Abbreviations: MISSILE: Metabolome Identification by Systematic Stable Isotope Labeling Experiments; nUPLC-HRMS: Nanoscale Ultra-Performance Liquid Chromatography-High Resolution Mass Spectrometry

\section{Introduction}

We recently described a formula-centric strategy for the identification of metabolites in untargeted metabolomics experiments [1,2]. The method uses stable isotope labeling [3-5] to identify the formula and structure of metabolites in an automated fashion with support for false discovery rate estimation [6,7]. Here we have expanded on this strategy by adding a fourth isotope labeling condition to enable 4-plex relative quantification of metabolites in LC-MS experiments. Each label represents an independent experimental condition so that we can compare the relative levels of metabolites from pairwise duplicates, time course, or dose-response experimental designs. Multi-plexed analyses offer many advantages over traditional label-free methods [8], but current strategies rely on specific functional group tags to chemically label metabolites [9] which limit the scope of quantified metabolites and introduce complexity to the sample preparation workflow. As a test of our methodology, we performed a dose-response analysis on the effect of the proteasome inhibitor Bortezomib [10] in yeast cells. This drug is an effective therapy for multiple-myeloma, but drug-resistance invariably develops due to incompletely characterized mechanisms $[11,12]$. The effect of proteasome inhibition on protein [13] and gene regulation [14] has been studied in a variety of contexts, but much less is known about the role of metabolism in this process. We report our preliminary investigation and demonstrate the analytical tools for carrying out multiplexed metabolomics analyses.

\section{Methods}

Materials-LC-MS grade acetonitrile (ACN), water, and formic acid (Sigma), glass beads (Next Advance), Difco ${ }^{\mathrm{TM}}$ yeast nitrogen base, DMEM (BD Biosciences), nanoLC column (Waters), ${ }^{13} \mathrm{C}-6$ glucose, ${ }^{15} \mathrm{~N}-2$ ammonium sulfate, (Cambridge Isotope Laboratories).

\section{MISSILE protocol}

For Saccharomyces cerevisiae (Fleischmann) labeling, cells were grown in four different minimal media conditions. A control media consisted of natural isotopic abundance components; Difco ${ }^{\mathrm{TM}}$ yeast nitrogen base without amino acids and ammonium sulfate (BD Biosciences), with $5 \mathrm{~g} / \mathrm{L}$ ammonium sulfate (Sigma), and $20 \mathrm{~g} / \mathrm{L}$ glucose (Sigma). For carbon-13 labeling, the media remained the same except that ${ }^{13} \mathrm{C}-6$ glucose (Cambridge Isotope Laboratories) was used in place of standard glucose. Similarly for nitrogen-15 labeling, ${ }^{15} \mathrm{~N}-2$ ammonium sulfate was substituted into the media. Each culture was maintained for $\sim 30$ generations in the labeled media before drug treatment and metabolite extraction. For Bortezomib treatment, cultures were seeded to an $\mathrm{OD}_{600}$ of 0.1 and allowed to grow to 1 ( $\sim 6$ generations).

\section{Bortezomib treatment}

Bortezomib was solubilized in $10 \mathrm{mM}, 1 \mathrm{mM}$, and $0.1 \mathrm{mM}$ stock solutions (water) so that equal volumes could be spiked in for each treatment. For each $25 \mathrm{~mL}$ yeast culture, the $\mathrm{OD}_{600}$ was monitored and recorded every hour. Bortezomib was added to each culture at $\mathrm{OD}_{600}=0.5$ absorbance units.

\section{Sample preparation}

Control and drug treatment yeast cultures were harvested and their metabolites were extracted. The liquid cultures were transferred to $15 \mathrm{~mL}$ conical vials and centrifuged at $1,000 \mathrm{~g}$ for $3 \mathrm{~min}$ to obtain

*Corresponding author: Jones DR, Department of Biochemistry and Molecula Pharmacology, Langone Medical Center, New York University, 550 1st Avenue, NY, 10016, USA, Tel: 646-501-2097; E-mail: Drew.Jones@nyumc.org

Peng J, Department of Structural Biology, St. Jude Children's Research Hospital, 262 Danny Thomas Place, Memphis, TN 38105, USA, Tel: (901) 595-7499; Fax: (901) 5953032; E-mail: Junmin.Peng@stjude.org

Received February 06, 2017; Accepted April 06, 2017; Published April 12, 2017

Citation: Jones DR, Wang X, Shaw T, Cho JH, Peng J (2017) Multiplexed Quantification of Metabolites with MISSILE. Metabolomics (Los Angel). 7: 189. doi:10.4172/21530769.1000189

Copyright: @ 2017 Jones DR, et al. This is an open-access article distributed under the terms of the Creative Commons Attribution License, which permits unrestricted use, distribution, and reproduction in any medium, provided the original author and source are credited. 
a cell pellet. The supernatant was discarded and $1 \mathrm{~mL}$ of freezing $80 \%$ acetonitrile was added. Each vial was subjected to $3 \mathrm{~min}$ of vortexing at $3,000 \mathrm{rpm}$ in a $1 \mathrm{on} / 1$ off pattern to maintain sample temperature [2]. The lysate was transferred to a fresh vial to exclude the glass beads, and then centrifuged at $21,000 \mathrm{~g}$ for $5 \mathrm{~min}$ to clarify the liquid phase.

\section{Spectrophotometric determination of multiplex mixing ratio}

The absorption $(300 \mathrm{~nm})$ of each supernatant was measured in a 1 $\mathrm{mL}$ cuvette and used to calculate the mixing ratio of the 4 individual cultures. For each culture we multiplied the absorbance reading by the volume of supernatant recovered $(900 \mu \mathrm{L})$ as an estimate of the total amount of metabolites present. We then calculated the amount of each sample needed to equal $90 \%$ of the lowest measured label. Using these values we mixed the supernatants of the 4 labeled samples into a single tube $(\sim 3 \mathrm{~mL})$, vortexed, and aliquoted the mixture into 4 separate tubes.

\section{Sample reconstitution}

The mixed or individual aliquots were dried under centrifugal vacuum and resolubilized to $75 \mu \mathrm{L}$ in buffer $\mathrm{A}$ and transferred to inserts for LC-MS analysis.

\section{LC-MS analysis and parameters}

Bortezomib treated yeast samples were analyzed on an Orbitrap Elite (Thermo Scientific) coupled to an Easy $\mathrm{nLC}^{\mathrm{TM}}$ system as previously described [2]. In summary, we used a nano Acquity UPLC column $(75 \mu \mathrm{m} \times 100 \mathrm{~mm})$ packed with $1.7 \mu \mathrm{m}$ BEH C18 beads with $0.2 \%$ formic acid in water (Mobile Phase A) or acetonitrile (Mobile Phase B). Sample injection volume was $2 \mu \mathrm{L}$. LC-MS analysis was performed in positive ion $(3 \mathrm{kV})$ mode with a $15 \mu \mathrm{m}, 5 \mathrm{~cm}$ PicoTip emitter (New Objective). A top 5 data-dependent method was used to target ions for fragmentation (MS/MS) for later structural identification.

\section{Results and Discussion}

\section{Proteasome inhibition of yeast cells and LC-MS analysis}

Yeast cells in a respiring liquid culture were subjected to proteasome inhibition by addition of Bortezomib. We treated cells with three concentrations of drug; at the reported $\mathrm{IC}_{50}$ [15], 10-fold below, and 10 -fold above, with a no-drug control (Figure 1a). To distinguish these four experimental conditions, carbon (i.e., glucose) and nitrogen (ammonium sulfate) sources in the culture were exchanged for various heavy stable isotope labeled compounds (i.e., ${ }^{13} \mathrm{C}$-6-glucose and ${ }^{15} \mathrm{~N}$-ammonium sulfate). There was a decrease in the yeast growth rate with increasing concentrations of Bortezomib (Figure 1b). We subjected each condition to LC-MS analysis alone (Figure 1c) or multiplexed (mixed together) and observed changes in the base-peak chromatogram due to drug-treatment.

\section{Formula and structure identification of Bortezomib regulated metabolites}

We used JUMPm software to globally analyze the metabolites from yeast cells treated with Bortezomib. As an example, we manually examined one of the down-regulated metabolites (a phosphatidylethanolamine). The MS1 scan shows four ions of varying mass, reflecting total incorporation of heavy stable isotopes into the chemical structure (Figure 1d). The mass shift of the four ions can also be used to determine the chemical formula of the metabolite (Figure 1e) as previously described. Each of the four parent ions for phosphatidylethanolamine was manually extracted to confirm their coelution and identity as isotope labels (Figure 1f). The intensity of these peaks was detected by JUMPm and serves as the basis for the relative quantification. The structure identity was determined by JUMPm in a search of its associated MS/MS spectra (Figure 1g). These tandem mass spectra were collected in a Top 5 data-dependent fashion. For phosphatidylethanolamine, MS2 spectra were acquired from two of the four labeled parent ions, the ${ }^{12} \mathrm{C}$ parent (unlabeled control) and the double labeled ${ }^{13} \mathrm{C}^{15} \mathrm{~N}$ parent ion. Two structural fragments were observed for each parent with nearly identical relative intensities. Overall, we identified 181 metabolite formulas and quantified 95 metabolite structures among the four treatment conditions with a false discovery rate of less than $1 \%$ (Table 1 ).

\section{Multiplexed quantification of Bortezomib regulated metabolites}

We observed that higher Bortezomib concentrations had broader impacts on the yeast metabolic profile, with a greater proportion of metabolites showing a larger fold change in peak size relative to control (Figure 1i). The lowest concentration of drug had a relatively minor effect on metabolite levels, whereas the higher concentrations increasingly segregated metabolites into up- or down-regulated populations. This trend was also true for individual metabolites (Figure 1d). As expected we observed that Bortezomib treatment increased the levels of incomplete protein catabolites e.g., di-peptides (Figure 1j). Metabolites that were unaffected by drug-treatment included nucleo-bases (e.g. adenine) and other "housekeeping" metabolites such as glutathione. We also observed that membrane components and signaling lipids were strongly down-regulated with higher Bortezomib concentrations (Figure $1 \mathrm{l}$ and 1k; Table 1).

\section{Advantages and limitations}

The described multiplexed design has several advantages over classical unlabeled strategies for metabolite quantification. Peak alignment between analyses is a major confounding factor for unlabeled strategies, and much effort has been focused on resolving this issue. For multiplexed samples, peak alignment is unnecessary because the treatment conditions are analyzed simultaneously. Carbon and nitrogen stable isotope labeling does not affect the retention time of small molecules, so each labeled form co-elutes with the other treatment conditions. This co-elution is a major advantage because it controls for time and sample-dependent variations in retention time and ionization efficiency, a major source of technical error during metabolite quantification.

Using the current strategy we can expect to identify and quantify metabolites from four independent labeled conditions. The labels are comprised of light or heavy carbon and nitrogen atoms. Therefore we observe all four possible mass labels for a given metabolite if the chemical formula contains at least one nitrogen atom. We previously observed that $\sim 50 \%$ of known metabolite formulas contain nitrogen while the rest do not. For metabolites without nitrogen, our strategy can only provide two independent labels. In that case, the two peaks represent the average of two cultures (i.e., cultures $1 \& 2$ or $3 \& 4$ ). Therefore the duplicate experimental design may be more suitable for studies focused on such metabolites.

We introduce a new capability to perform 4-plex relative quantification of metabolites in untargeted metabolomics experiments using the MISSILE strategy in combination with JUMPm software. Using this method, we can now analyze metabolites from two experimental conditions with duplicates, or with up to 4 independent experimental conditions (e.g. time series or dose response data). The 
a
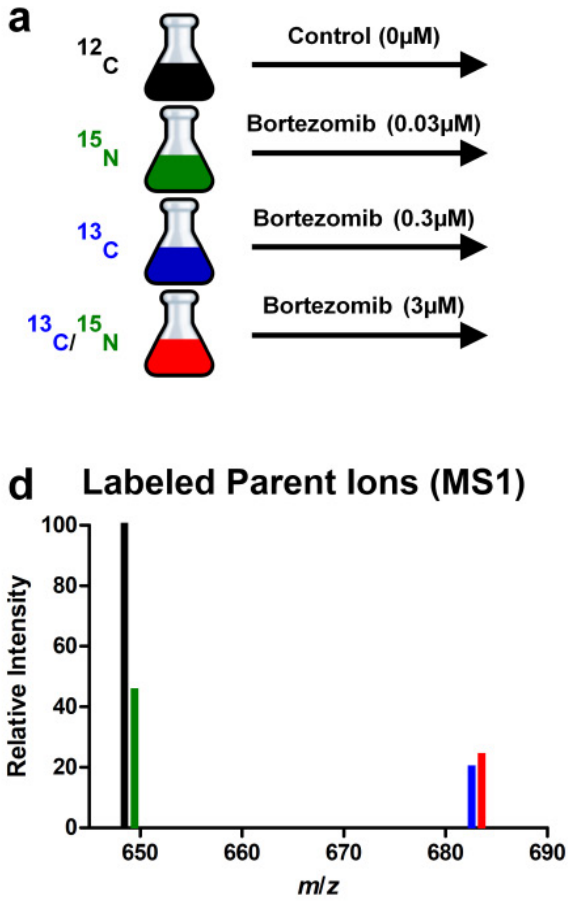

g Labeled Fragment Ions (MS2)
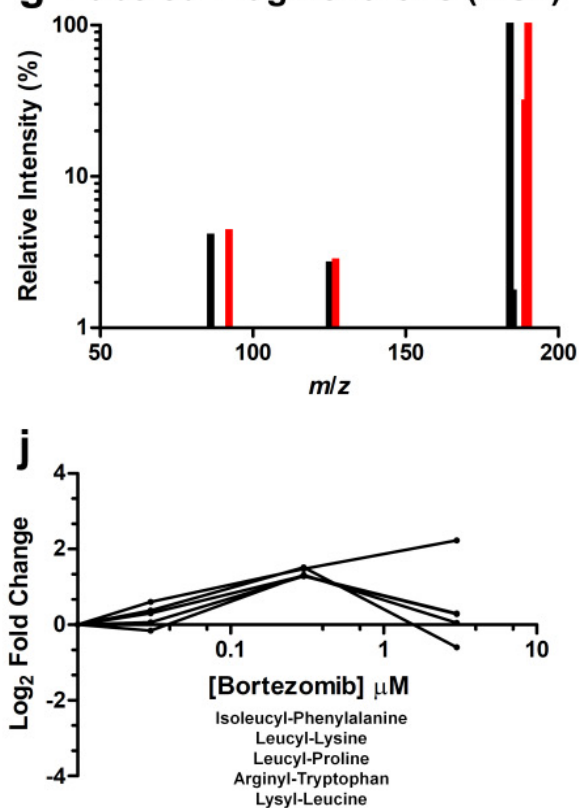

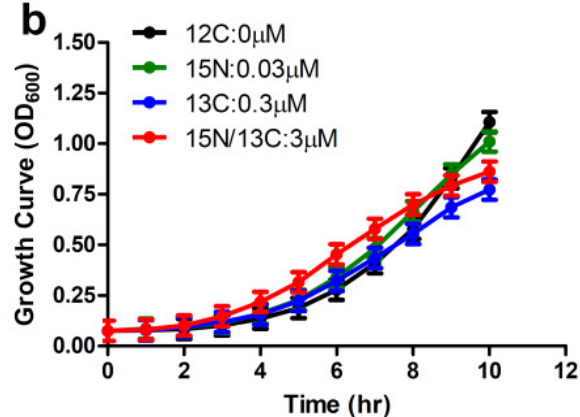

e $\quad 648.4615 \quad \mathrm{C}_{34} \mathrm{H}_{2} \mathrm{~N}_{1} \mathrm{O}_{?} \mathrm{~S}_{2} \mathrm{P}_{2}$ $z=1$

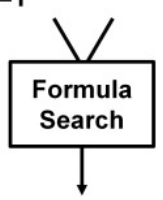

Candidates by mass 165 Candidates by mass, \#C 10 Candidates by mass, \#C, \#N 1 $\left[\mathrm{C}_{34} \mathrm{H}_{67} \mathrm{~N}_{1} \mathrm{O}_{8} \mathrm{P}_{1}\right]^{+}$

h
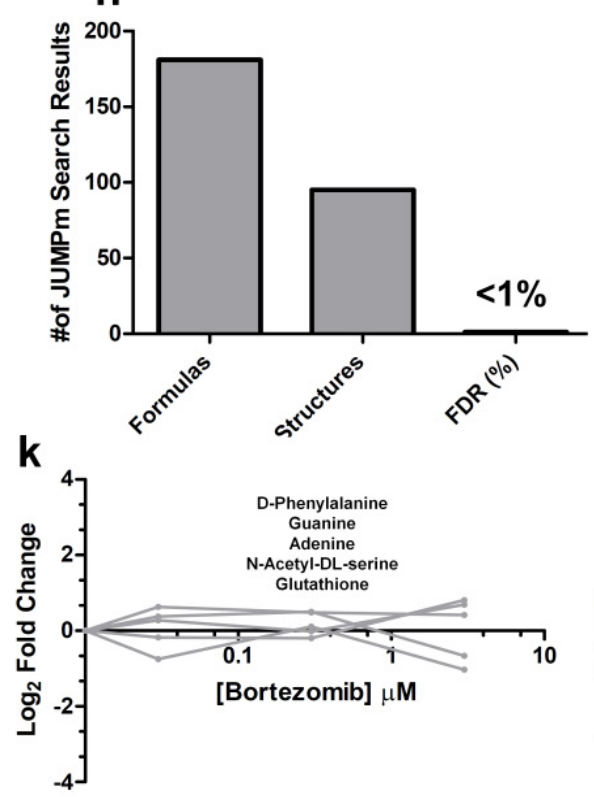

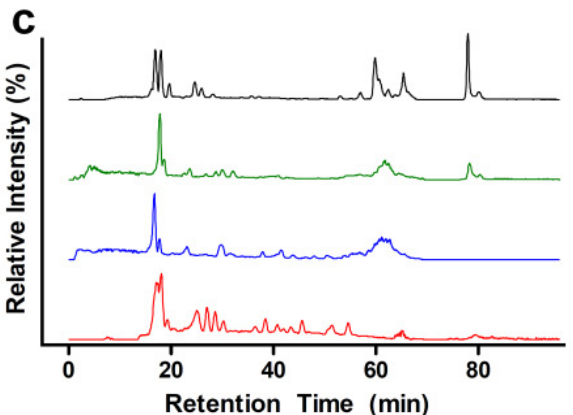

f Extracted Labeled lons

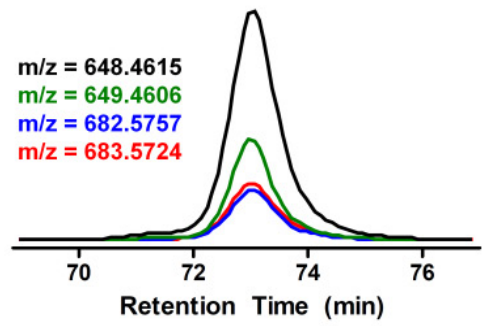

i

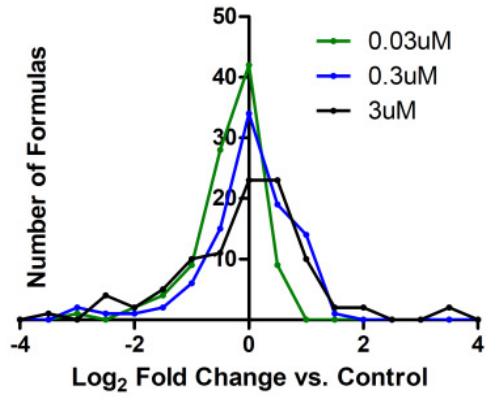

I

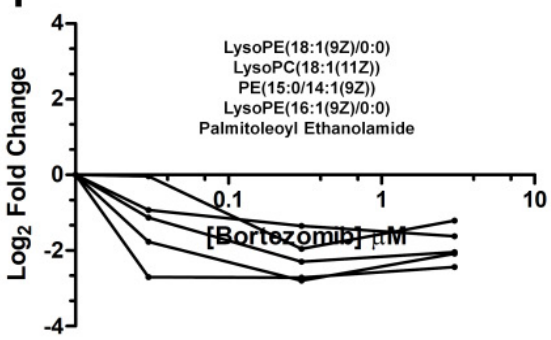

Figure 1: Metabolomics analysis of Bortezomib treated yeast cells (a) Experimental design of the 4-plex study. Labels indicate the stable isotope culture condition. Condition 4 (red) used both stable isotope labels simultaneously. (b) Growth curve for the four independent yeast cultures, monitored by OD chromatograms for the four cultures analyzed independently prior to multiplex analysis. (d) An example MS1 spectrum for a metabolite peak after mixing the four yeast samples together. Colored peaks reflect the increased mass of the metabolite from stable isotope labelling and also the relative intensity changes due to Bortezomib treatment. This metabolite was identified as PE (15:0/14:1(9Z)). (e) Automated chemical formula-determination workflow for the example metabolite peaks shown in (d). Candidates represent chemical formulas within $4 \mathrm{ppm}$ mass tolerance of the unlabelled peak. With increasingly strict search criteria the number of possible formulas dramatically decreases. The determined formula represents the charged parent ion. All formulas in Table 1 have been discharged. (f) Extracted ion chromatogram for each of the four labeled versions of the yeast metabolite shown in (d). Retention time and peak shape are not affected by isotope labelling, while the peak intensity reflects the changes due to Bortezomib treatment. (g) Tandem mass spectrum (MS/MS) of the labeled precursors from the example metabolite. (d-f) Each structure fragment shows two peaks, representing the two isotopes labeled parent ions which were fragmented in the Top 5 data-dependent method. ( $h$ ) Fold change distribution of quantified metabolites compared to the no-drug control. Higher Bortezomib concentrations were associated with increased variation in metabolite levels. (i) Summary of JUMPm global metabolite search results. The formula false discovery rate (FDR) was less than $1 \%$ as determined by JUMPm based on the relative frequency of target and decoy formulas detected. (j-I) Up-regulated, unaffected, and down-regulated metabolites respectively as a function of Bortezomib concentration. Labels indicate identified metabolites using JUMPm software. 


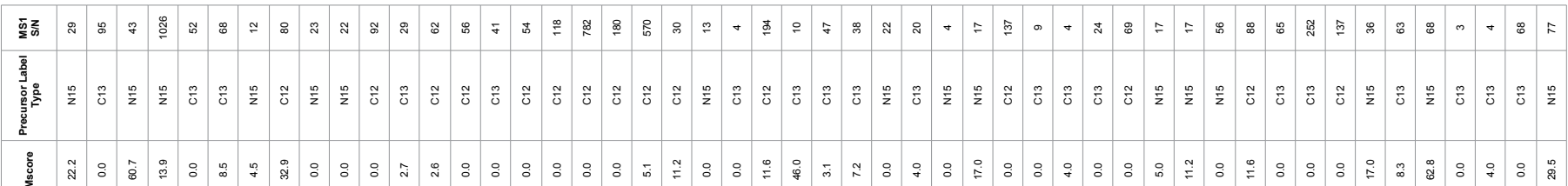

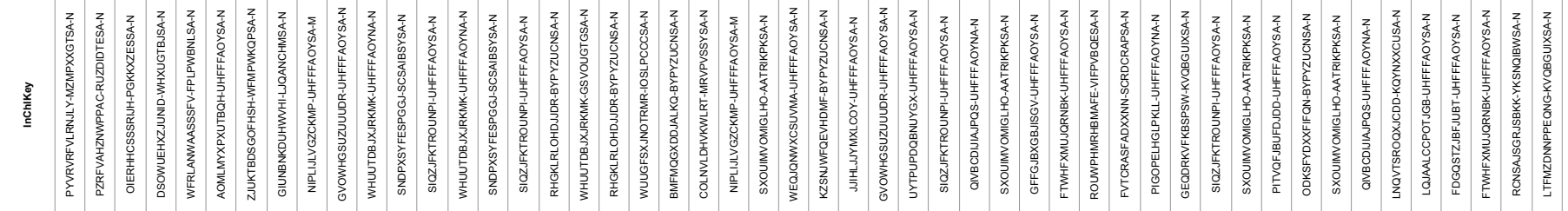

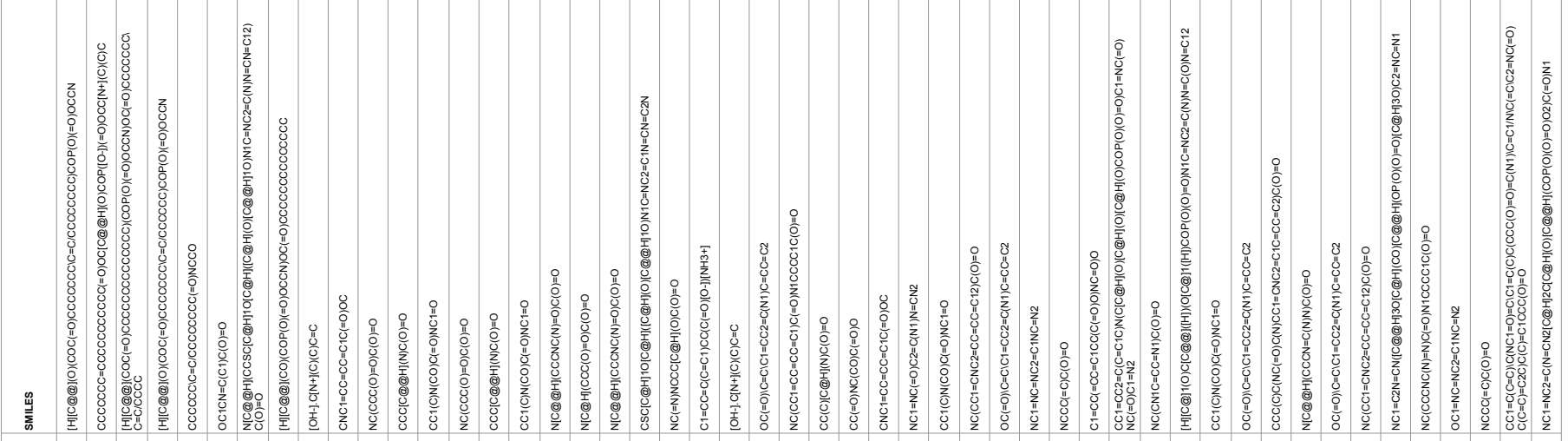

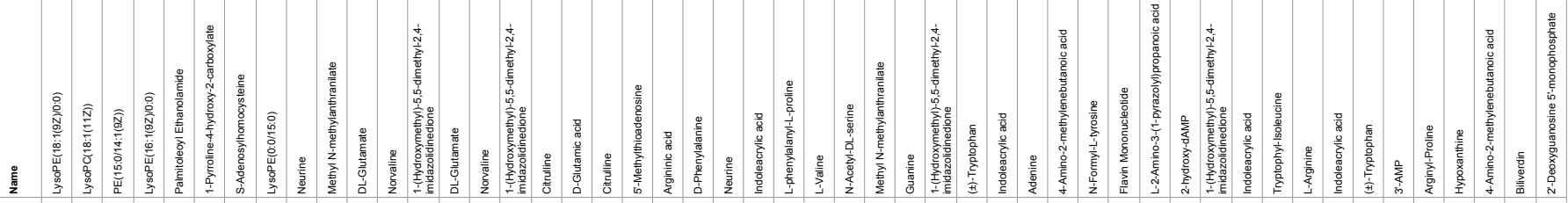

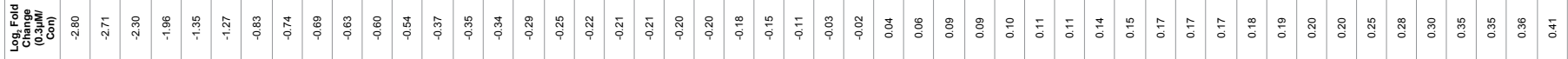

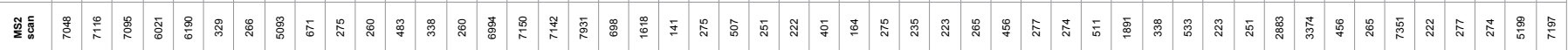

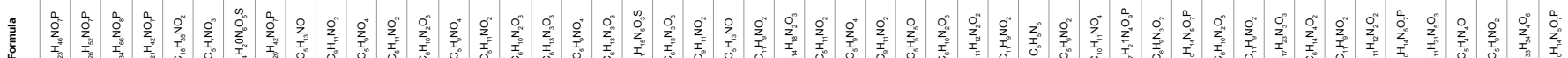

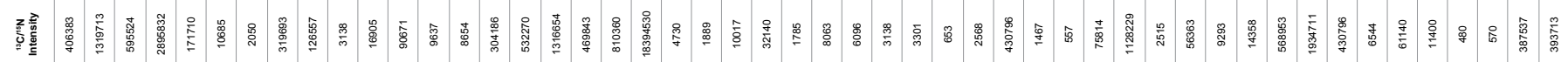

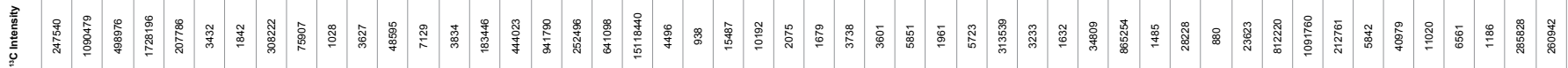

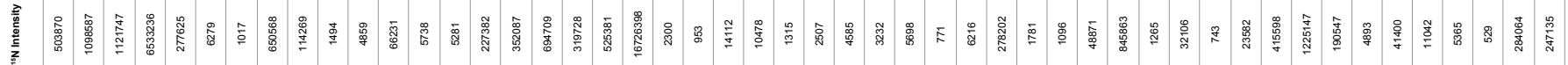

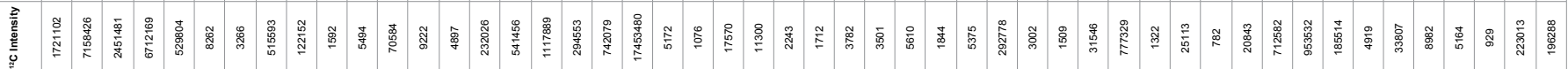

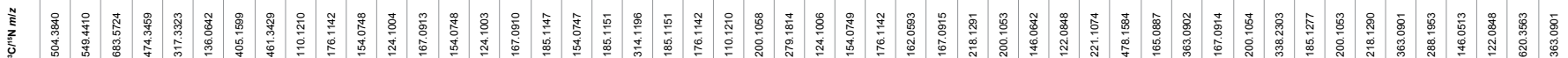

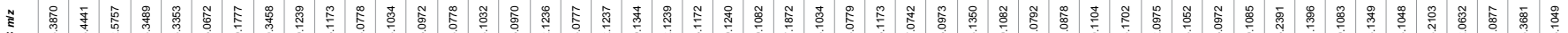

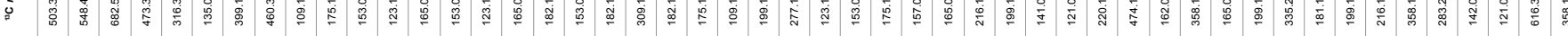

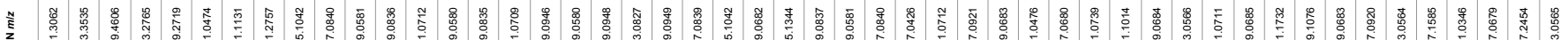

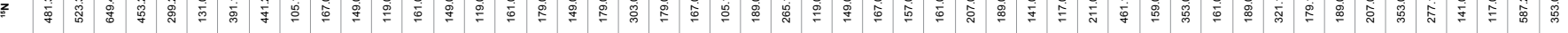

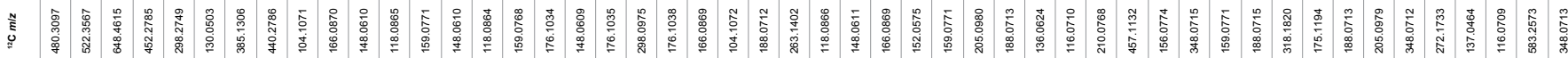

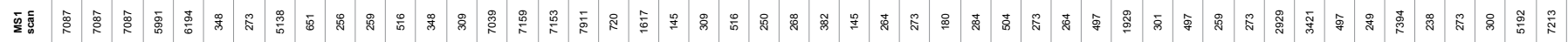


Citation: Jones DR, Wang X, Shaw T, Cho JH, Peng J (2017) Multiplexed Quantification of Metabolites with MISSILE. Metabolomics (Los Angel). 7: 189. doi:10.4172/2153-0769.1000189

Page 5 of 6

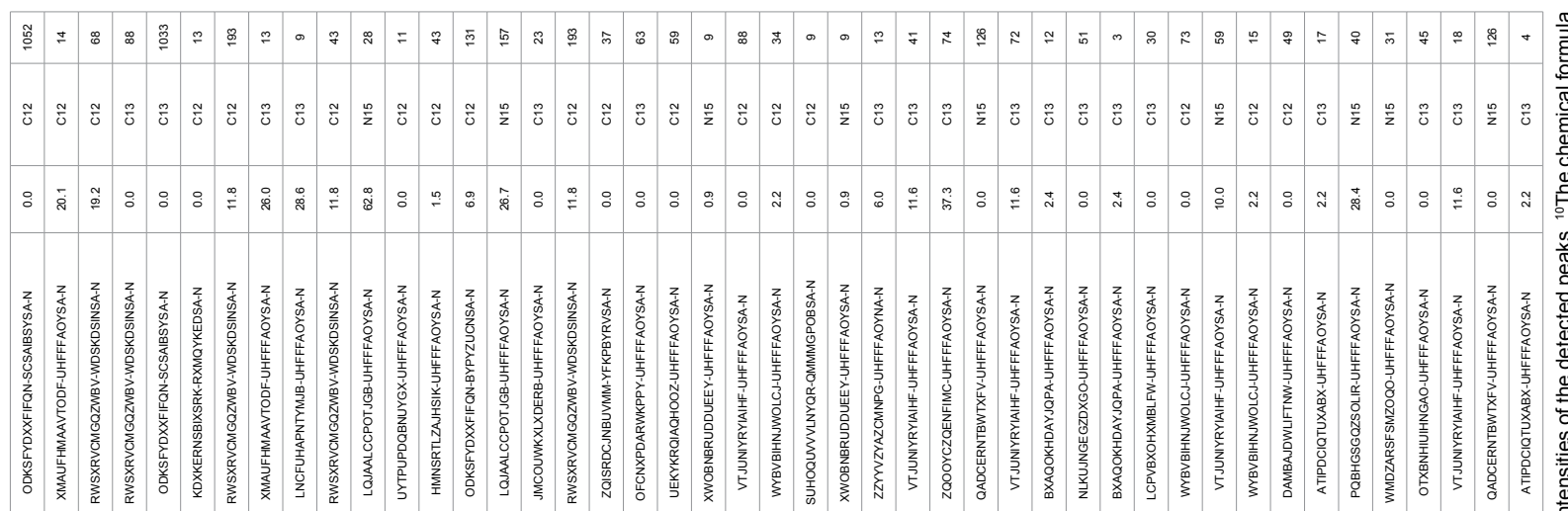


Citation: Jones DR, Wang X, Shaw T, Cho JH, Peng J (2017) Multiplexed Quantification of Metabolites with MISSILE. Metabolomics (Los Angel). 7: 189. doi:10.4172/2153-0769.1000189

Page 6 of 6

isotope labels are used by JUMPm software to determine chemical formulas and perform relative quantification and false discovery control.

\section{Acknowledgements}

The authors thank Z. Wu for laboratory assistance, H. Tan, A. High and V. Pagala for MS instrument guidance, and other lab and facility members for helpfu discussion. This work was partially supported by National Institutes of Health grants R01GM114260, R01AG047928, R01AG053987, R01AG047928, R01GM114260 the American Asthma Foundation (15-0020), and ALSAC (American Lebanese Syrian Associated Charities). The MS analysis was performed in the St. Jude Children's Research Hospital Proteomics Facility, partially supported by NIH Cancer Center Support Grant (P30CA021765).

\section{References}

1. Jones DR, Shaw XWT, Cho JH, Chen PC, Peng J, et al. (2016) Metabolome Identification by Systematic Stable Isotope Labeling Experiments and False Discovery Analysis with a Target-Decoy Strategy. Biorxiv.

2. Jones DR, Wu Z, Chauhan D, Anderson KC, Peng J (2014) A Nano UltraPerformance Liquid Chromatography-High Resolution Mass Spectrometry Approach for Global Metabolomic Profiling and Case Study on Drug-Resistant Multiple Myeloma. Anal Chem 86: 3667-3675.

3. Giavalisco P, Kohl K, Hummel J, Seiwert B, Willmitzer L (2009) 13C isotopelabeled metabolomes allowing for improved compound annotation and relative quantification in liquid chromatography-mass spectrometry-based metabolomic research. Anal Chem 81: 6546-6551.

4. Giavalisco P, Li Y, Matthes A, Eckhardt A, Hubberten HM et al. (2011) Elemental formula annotation of polar and lipophilic metabolites using (13) C, (15) $\mathrm{N}$ and (34) $\mathrm{S}$ isotope labelling, in combination with high-resolution mass spectrometry. Plant J 68: 364-376.

5. Hegeman AD, Schulte CF, Cui Q, Lewis IA, Huttlin EL, et al. (2007) Stable isotope assisted assignment of elemental compositions for metabolomics. Anal Chem 79: 6912-6921.

6. Elias JE, Gygi SP (2007) Target-decoy search strategy for increased confidence in large-scale protein identifications by mass spectrometry. Nat Method 4: 207-214.

7. Peng J, Schwartz D, Elias JE, Thoreen CC, Cheng D, et al. (2003) A proteomics approach to understanding protein ubiquitination. Nat Biotechnol 21: 921-926.

8. Ong SE, Blagoev B, Kratchmarova I, Kristensen DB, Steen H, et al. (2003) Stable isotope labeling by amino acids in cell culture, SILAC, as a simple and accurate approach to expression proteomics. Mol Cell Proteomics 1: 376-386.

9. Su X, Wang N, Chen D, Li Y, Lu Y, et al. (2016) Dansylation isotope labeling liquid chromatography mass spectrometry for parallel profiling of human urinary and fecal submetabolomes. Anal Chim Acta 903: 100-109.

10. Field-Smith A, Morgan GJ, Davies FE (2006) Bortezomib (Velcadetrade mark) in the Treatment of Multiple Myeloma. Therapeutics and Clinical Risk Management 2: 271-279.

11. Murray MY, Auger MJ, Bowles KM (2014) Overcoming bortezomib resistance in multiple myeloma. Biochemical Society Transactions 42: 804-808.

12. Lu S, Wang J (2013) The resistance mechanisms of proteasome inhibitor bortezomib. Biomark Res 1: 13.

13. Dytfeld D, Rosebeck S, Kandarpa M, Mayampurath A, Mellacheruvu D, et al. (2015) Proteomic profiling of naive multiple myeloma patient plasma cells identifies pathways associated with favourable response to bortezomib-based treatment regimens. $\mathrm{Br} \mathrm{J}$ Haematol 170: 66-79.

14. Stessman HA, Baughn LB, Sarver A, Xia T, Deshpande R, et al. (2013) Profiling bortezomib resistance identifies secondary therapies in a mouse myeloma model. Mol Cancer Ther 12: 1140-1150.

15. Huber EM, Heinemeyer W, Groll M (2015) Bortezomib-resistant mutant proteasomes: structural and biochemical evaluation with carfilzomib and ONX 0914. Structure 23: 407-417. 